https://ojs.unud.ac.id/index.php/jbn

\title{
Fournier Gangrene in A 65 Years Old Obese Female with Uncontrolled Type II Diabetes Mellitus - A Case Report
}

\author{
Rochella Krismurning Coffee, Kelvin Setiawan*, Bramastha Aires Rosadi, Terry Renata \\ Lawanto
}

Department of Surgery, Tebet General Hospital, South Jakarta, Indonesia

*Corresponding author: kelvin.setiawan.a@gmail.com.

\begin{abstract}
Background: Fournier gangrene is a disease with characteristic of rapidly progressive necrotizing fasciitis in the perianal and genitourinary area. Case: Usually it affects men, but we hereby present Fournier gangrene in an obese, diabetic, middle-aged woman. Her chief complaint was discomfort, swelling and foul odor coming from her genital area. Thus, she was diagnosed with Fournier gangrene and underwent emergency surgical debridement with local anaesthesia. After removing all necrotic tissues, overlying pus and debris, wounds were left open until granulation were seen and optimal condition of the tissue and planned for reconstructive surgery and additional debridement if needed. Local wound dressings were done with silver dressings and washed with $0.1 \%$ Polyaminopropyl biguanide solution. Patient received oral antibiotics daily for five days and visit surgery polyclinic twice a week for wound toilet and observation of wound condition. Conclusion: Early diagnosis, administration of broad-spectrum antibiotics and emergency debridement is important factors to successful outcome of Fournier gangrene.
\end{abstract}

Keywords: diabetes mellitus type II, female, fournier gangrene, modern wound dressing, necrotizing fasciitis.

DOI: https://doi.org/10.24843/JBN.2020.v04.i01.p01

\section{INTRODUCTION}

Fournier gangrene, necrotizing fasciitis of the perineal, genital and perianal area, is one dangerous disease and life-threatening, as it can spread to the abdominal wall, causing soft tissue necrosis and eventually lead to sepsis. $^{1} \quad$ Fournier gangrene was first discovered and described by Baurienne in 1764, but it was named after Jean-Alfred Fournier - a French venerology's, who reported it in 1883 as a rapidly progressive or fulminant genital gangrene in otherwise healthy young men, with a sudden onset and no apparent cause or specific etiology. ${ }^{2}$ This condition is relatively rare, with an estimated overall incidence of 1.6 / 100,000 males. The disease typically affects and predominates in males, but rarely Fournier Gangrene is also diagnosed in woman. ${ }^{3}$ Patients with Fournier Gangrene usually have medical or surgical conditions, which are predisposing factors to this disease or it's more severe or fatal course. These risk factors consist of hypertension, diabetes mellitus, advanced age, alcoholism, and poor general health, including chronic renal failure, chronic liver disease, malnutrition or obesity, malignancies and other immunodeficiency conditions. ${ }^{2}$ In this occasion, we hereby present a Fournier gangrene in a female patient, 65-year-old with diabetic and obese body mass index.

\section{CASE REPORT}

A female obese patient aged 65 years old from Tebet, Jakarta, was referred to surgical department in Tebet General Hospital, 
Jakarta from obstetric and gynaecologic and oxygen saturation. Her body mass index department with complains of swelling and was $34.1 \mathrm{~kg} / \mathrm{m} 2$. Her weight was $82 \mathrm{~kg}$ and ulceration in her genital region. Swelling and $155 \mathrm{~cm}$ height tall. The physical examination ulceration was seen in her right vaginal major revealed foul odor oedematous right-side labia. Patient was unable to describe initial findings. Patient did not feel any significant pain, but experiencing fever 2 weeks before her admission and foul odor from her genital area.

She experienced vaginal asymmetrical inflammatory swelling and fever without any urinary symptoms, abdominal pain, changes in the bowel habits or vaginal and rectal bleeding. The swelling became more prominent and patient started to feel uncomfortable when tried to sit down. Patient had a history of uncontrolled type II diabetes mellitus with blood glucose level of 400 $\mathrm{mg} / \mathrm{dl}$ in her first laboratory findings when admitted. Patient usually control her blood glucose level monthly in public health care near her house and taken oral medications such metformin $500 \mathrm{mg}$ three times daily and glipalamide $1 \mathrm{mg}$ once daily. Patient also had a history of poor genital hygiene.
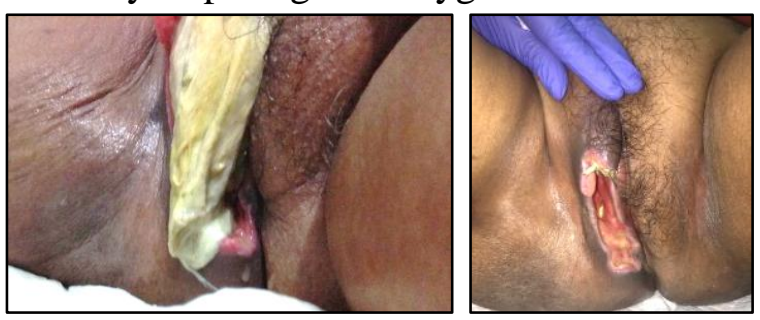

Figure 1. Patient's first admission on polyclinic (left) and after debridement (right).

Patient felt really uncomfortable with swelling on the vaginal and foul odor, thus decided to visit obstetric and gynaecologic department in Tebet General Hospital. The gynaecologist then referred the patient to surgery department in Tebet General Hospital. She appeared mildly sick, not in pain and had difficulty when walking. She had a body temperature of $37.5^{\circ} \mathrm{C}$, and normal value of blood pressure, heart rate, labia majora skin, covered with pus in the centre and necrotic skin around the lesion (Figure 1 left). The swelling extended into medial side of the right thigh with minimal pain and crepitations or haemorrhagic was not found from this lesion. The initial laboratory investigations had remarkable value for a haemoglobin of $9,8 \mathrm{mg} / \mathrm{dl}$, and white blood cell of $15,000 \mathrm{~g} / \mathrm{dl}$ with $75 \%$ neutrophils.

In the outpatient surgery clinic, initial debridement, wound toilet and removal of necrotic skin were done. Patient was positioned into lithotomy position and was given local anaesthetic, which was lidocaine $40 \mathrm{mg}$ around the lesion. Necrotic skin and debris were removed from the lesion, and planned to do surgical sharp debridement with curettage. At the base of the lesion, we found significant amount of discharge with brown colour, foul smell and surrounded with necrotic tissue on the edge. The wound appeared to be deeper than predicted when the wound was cleared for any debris and pus at the centre (Figure 1 right). We measure the depth of infiltrating injury with index finger and found no openings into genitourinary tracts and into intestine.

The wound then was cleaned with $0.1 \%$ Polyaminopropyl biguanide solution and silver wound dressings on top of the debrided lesion. The wound was deliberately stayed open until the infections were fully controlled and planned to close the wound with local skin flaps in the operating room. Patient was given oral antibiotics, ciprofloxacin $500 \mathrm{mg}$ twice daily and oral analgesics, paracetamol $500 \mathrm{mg}$ three times daily for five days. Patient was taught of genital hygiene and wound toilet at home, and encouraged to 
continue her DM medication. Patient then emerging from the wound in patient's last discharged home and had to visit surgery visit to our clinic. The granulation gradually polyclinic two times a week for wound toilet, and passively closed the deep surface of the wound observation, and plans of wound wound and infections appeared no longer closure. active. Patient then was decided to close the

Patient visited our outpatient clinic six wound in operating room with local skin times in three weeks, and showed good result flaps. Anaesthesia teams were consulted for (Figure 2). The production of purulent preoperative evaluations and mandatory discharge became less and finally stopped laboratory tests were done.
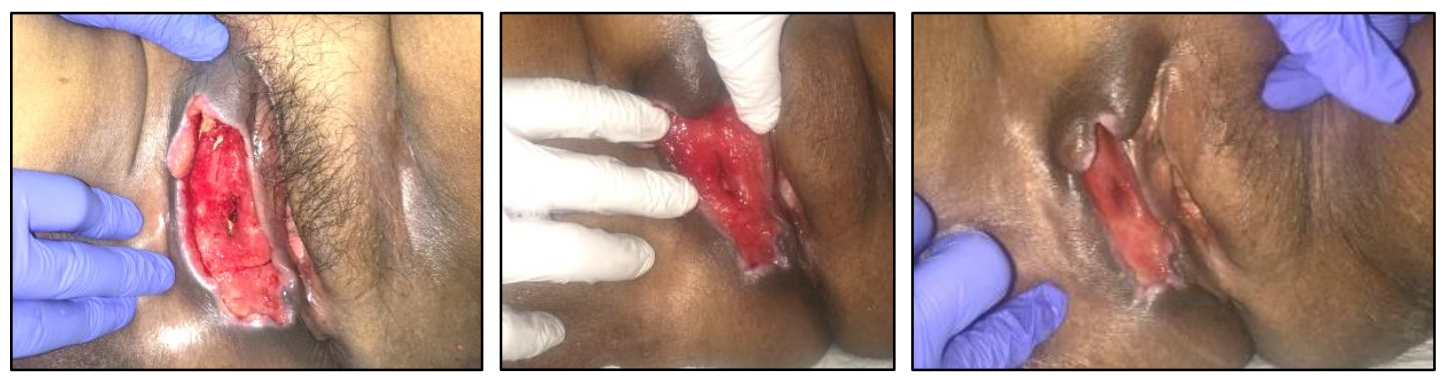

Figure 2. Process of wound healing with modern wound dressings in three weeks.

In the operating room, under general anaesthesia, patient was put into lithotomy position (Figure 3 left). We examined the wound and decided not to do another surgical debridement, due to decent wound appearance with no other debris and necrotic tissues. We washed the wound with normal saline solution and closed the wound with simple interrupted sutures. After closure, the wound was closed with sterile moist dressings and transferred to hospital ward for observation after operation (Figure 3 right).
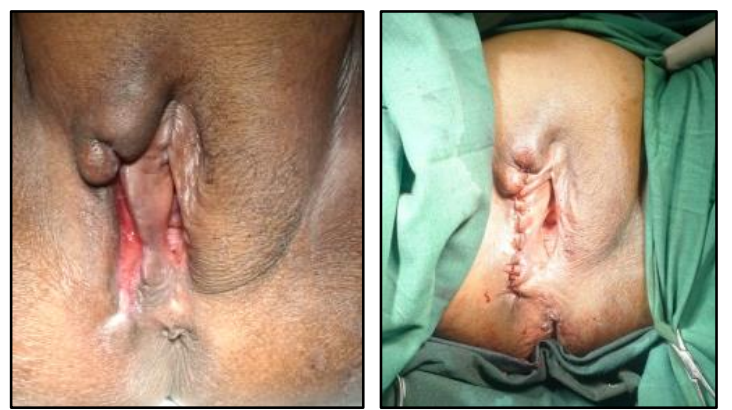

Figure 3. Condition of wound before primary closure (left) and after primary closure in the operating room (right).

Patient was given oral analgesics, dexketoprofen $25 \mathrm{mg}$ three times daily and oral antibiotics, ciprofloxacin $500 \mathrm{mg}$ two times daily for five days. After waking up from general anaesthesia, patient was observed for complications postoperatively and eventually discharged home after 24 hours of hospital admission and advised patient to routinely follow-up at an outpatient surgery clinic for surgical wound control.

\section{DISCUSSION}

As discussed above, Fournier gangrene is one of the life-threatening diseases, caused by necrotizing fasciitis of the genitalia region, which can spread locally and invasively. It was first originally described by Baurienne in 1764 and by Fournier in 1883, Fournier gangrene was characterized by an immediate onset of a rapidly fulminating gangrene in genital area, which emerged as idiopathic lesion in previously young and healthy patients, and resulted in inflammatory destruction of the genitalia. ${ }^{4}$ Although Fournier previously described this disease as an idiopathic origin, in fact Fournier gangrene is rarely truly idiopathic and with 
careful examination and observation, an underlying and risk factors contributing to the disease can be identified in most cases. ${ }^{3}$ In our reports, patient did not notice any changes in her genital, until patient found a foul odor coming from her genital area and experienced fever and discomfort. Whether this process was idiopathic or there was an underlying cause, it is still unclear due to lack of attention from the patient.

In the twentieth century, there was a change in the patient's age distribution towards older ages, contrary to Fournier's first description. Also, current understanding describes this disease as an illness which no longer limited to male gender, but it also affects female and even children. However, studies and reports regarding Fournier gangrene in female and children are very few. ${ }^{5}$ In this report, we already described one example of female patient with Fournier's gangrene. Diagnosis and treatment options are based on guidelines and updates regarding Fournier's gangrene in men. We have not found any updates and guidelines regarding Fournier's gangrene in female patient. Further study about this issue are needed to overcome any patient with same conditions.

Fournier gangrene tends to pose a threat to individuals with immunocompromised conditions, which means patient with systemic disorders such as diabetes mellitus are at increased risk of developing this disease. Prolonged hyperglycaemia serum has damaging effects on patient's immunity status via its adverse effect on phagocyte activity, chemotaxis and cellular adherence. Thus, as previously described, diabetes has a role as predisposing factor in $32 \%$ to $66 \%$ Fournier gangrene cases.3 In our report, patient had a history of diabetes mellitus type II and routinely checked her blood glucose level in public healthcare nearby. However, her blood glucose at her first admission to our hospital was $400 \mathrm{mg} / \mathrm{dl}$, indicating an uncontrolled diabetes mellitus. Another predisposing factor for Fournier gangrene are poor general health, including malnutrition or obesity, chronic renal failure, chronic liver disease, malignancies and other conditions causing immunosuppression. ${ }^{2}$ Our patient was obese with body mass index (BMI) of $34.1 \mathrm{~kg} / \mathrm{m} 2$, which is considered a contributing factor to the development of Fournier gangrene.

The classic symptoms of Fournier gangrene consist of swelling, erythema and scrotal pain (or vaginal pain in women). Systemic symptoms are often present such as fever, tachycardia and sepsis. Although it was first described as sudden onset disease, Fournier gangrene has shown to be indolent and progressive, as experience has shown. These symptoms such as pain, pruritus and discomfort tend to worsen with period of 3-5 days before hospital admission. The onset of the disease is more insidious, which result in delayed diagnosis and treatment in up to $40 \%$ of cases. $^{3}$ In our report, patient chief complaint was foul odor, discomfort in genital area, erythema and fever. Interestingly, patient didn't feel any significant pain in her genital. This may be caused by diabetic peripheral neuropathy, resulting in decreased of pain sensation in certain affected area. Delayed diagnosis and treatment also couldn't be avoided, due to late admission to the hospital, which explained that this patient also experienced an indolent onset, rather than a sudden and abrupt onset.

Treatment of this dangerous disease consists of fluid resuscitation, extensive debridement of dead tissues and excision of necrotic and devitalized tissue, and administration of broad-spectrum antibiotics. After these treatments are administered, some 
patient can be left with extensive tissue defects, which some of these cases need reconstructive procedures such as flaps, grafts or simple closure by suturing.

In a review of Fournier Gangrene Reconstructive Cases by Ferreira and colleagues, wide surgical debridement of scrotal, penile and perineal necrosis along with other involved areas were initially performed in all patients. All patients received broad spectrum antibiotics administered intravenously.

Local wound care was performed with moist gauze dressings (i.e., saline, potassium permanganate or Dakin's solution) changed two or three times per day until healthy granulation tissue was present; subsequently, dry gauze dressing was used. All patients in Ferreira and colleagues review received delayed surgical reconstruction after the appearance of healthy granulation tissue at the base of the wound. The mean time between the last debridement performed and the first reconstructive procedure was $37.4 \pm$ 23.6 days (range 12 to 115 days). ${ }^{7}$ In our report, patient underwent an immediate debridement. Removal necrotic skins, debris and overlying pus were the main objectives. Wound were left open until granulation were seen at the base and inner edge of the wound.

The time between the last debridement performed and the first reconstructive procedure in our case is 21 days. We provide also a local wound dressing using silver and $0.1 \%$ Polyaminopropyl biguanide solution as local wound care before reconstruction. We didn't use any dry gauze dressings, due to dry condition of the wound.

Patient received broad spectrum antibiotics orally and allowed to be treated at home. Patient did not show any signs of septic shock and we educated patient and her family about wound care at home. Without any fistula and other destruction of surrounding tissues and optimal conditions of primary wound, we decided not to undergo another debridement surgery and close the wound primarily with simple interrupted sutures.

\section{CONCLUSION}

Fournier gangrene is a rare disease, especially in woman as the reported incidence is $1.6 / 100,000 \mathrm{pf}$ the male population with male cases outnumbering female cases at a ratio of 10:1 and the highest incidence is persons aged 50 to 70 years. The predisposing factors to this disease include diabetes mellitus, hypertension, alcoholism, advanced age, malnutrition or obesity, chronic disease such as liver and renal disease and other malignancies or immunodeficiency conditions.

The clinical symptoms include pain in the genital area, swelling, erythema, discomfort, fever, pallor and fever. It is important to conclude that the keys for a successful outcome of Fournier gangrene include early diagnosis, rapid administration of broadspectrum antibiotics and immediate debridement.

\section{ACKNOWLEDGEMENT}

None.

\section{DISCLOSURE}

Authors declare no conflict of interest of this study.

\section{REFERENCES}

1. Althunayyan S, Karamitosos E. Fournier's gangrene in an obese female in third semester of pregnancy. Saudi Med J. 2018;39:415-8.

2. Wroblewska M, Kuzaka B, Borkowski T, et al. Fournier's gangrene - current concepts. Pol J Microbiol. 2014;63:26773. 
3. Singh A, Ahmed K, Aydin A, et al. 5. Czymek R, Frank P, Limmer S, et al. Fournier's gangrene. A clinical review. Fournier gangrene: is the female gender a Arch Ital Urol Androl. 2016;88:157-64. risk factor?. Langenbecks Arch Surg.

4. Schaeffer AJ, Scaeffer EM. Infections of 2010;395:173-80.

the Urinary Tract. In: Kavoussi LR, 6. Ferreire PC, Reis JC, Amarante JM, et al. Novick AC, Partin AW, et al, editors. Fournier gangrene: a review of 43 Campbell-Walsh Urology. $10^{\text {th }}$ edition. Philadelphia: Saunders; 2012. p.324-5. reconstructive cases. Plast Reconstr Surg. 2007;119:175-84. 